\title{
Laparoscopic-assisted Soave operation for the treatment of Hirschsprung disease in children: 5 years of experience
}

\section{Mingkun Liu}

Fujian Medical University

Yifan Fang ( $\nabla$ sfyfangyifan@163.com )

Fujian Medical University

Bing Zhang

Fujian Medical University

Yu Lin

Fujian Medical University

\section{Oumin Li}

Fujian Medical University

Jianxi Bai

Fujian Medical University

Dianming Wu

Fujian Medical University

\section{Research article}

Keywords: Laparoscope, the Soave operation, Hirschsprung disease in children

Posted Date: March 30th, 2020

DOl: https://doi.org/10.21203/rs.3.rs-18886/v1

License: (c) (i) This work is licensed under a Creative Commons Attribution 4.0 International License. Read Full License 


\section{Abstract}

Purpose: The purpose of this study was to summarize the clinical experience of the laparoscopic-assisted Soave operation for the treatment of Hirschsprung disease in children. Methods: In total, 186 children with Hirschsprung disease participated in this study from January 2014 to January 2019. The Soave operation was used to treat Hirschsprung disease with laparoscopic assistance. Symptoms and signs were followed up at one week, one month, three months, six months, one year and every 1-2 years after the first year. Results: All 186 children underwent laparoscopic surgery successfully, and none progressed to open surgery. During hospitalization and follow-up, there were 49 patients with complications, including 1 patient with an anastomotic leakage, 1 with an anal stricture, 5 with constipation recurrence, 5 with dirty defecation, 22 with enterocolitis, and 15 with perianal erosion. There were no complications such as abdominal bleeding, abdominal infection, ureter injury, adhesive intestinal obstruction, anastomotic stricture, incontinence. Conclusion: The laparoscopic-assisted Soave operation is a safe and feasible method for the treatment of Hirschsprung disease in children. This method has the advantages of less trauma and good cosmetic effects.

\section{Introduction}

Hirschsprung disease is one of the most common congenital digestive tract malformations in paediatric surgery, with an incidence of approximately $1 / 5000^{[1-3]}$. The main symptoms are delayed defecation, vomiting, progressive abdominal distension and constipation. If timely and effective treatment is not carried out, poor digestive system function can result, which affects growth and even leads to the death of children ${ }^{[4]}$. Surgical treatment is often needed in children with megacolon, and traditional surgical methods include the open operation of Duhamel, Swenson and Soave with great trauma ${ }^{[5,6]}$.

With the development and application of laparoscopic surgery in paediatric surgery and the continuous improvement of megacolon surgery, the operation for Hirschsprung disease has gradually evolved to laparoscopy-assisted combined with transanal anal pulling. As early as $1994^{[7]}$ and $1995^{[8]}$, Smith and Georgeson reported on the Duhamel and Soave operations assisted by laparoscopy. Subsequently, the Soave operation with laparoscopic assistance has been widely used in the clinic, and remarkable results

have been achieved ${ }^{[0-11]}$. We retrospectively analysed the clinical data of 186 children with Hirschsprung disease undergoing the Soave operation assisted by laparoscopy in our hospital over 5 years to summarize the experience and clinical efficacy of this surgical method.

\section{Methods}

\section{Patients}

We retrospectively analysed the clinical data of 186 children with Hirschsprung disease in our hospital from January 2014 to January 2019, including the preoperative, intraoperative, postoperative and followup data. Patients met the inclusion criteria if they underwent the Soave operation assisted by 
laparoscopy. Patients were excluded from this study if they 1) underwent other surgical methods; 2) had severe liver and kidney dysfunction and complex congenital heart disease; and 3) refused to sign the consent form for surgery or refused to comply with the follow-up schedule.

All the children had symptoms of delayed defecation and long-term or repeated constipation.

Hirschsprung disease was clearly diagnosed by the examination of transanorectal manometry, barium colonography, intraoperative frozen pathology and postoperative paraffin pathology, including 135 patients with the common type, 43 with the long segment type, and 8 patients with the total colon type. There were 122 males and 64 females. The age was from 2.3 months6.3 years old, and the weight was $3.5 \mathrm{~kg}-26.6 \mathrm{~kg}$ (Table 1). A routine clinical examination was performed before the operation, including an electrocardiogram, chest radiography, cardiac colour Doppler ultrasound and blood examination.

\section{Technology}

\section{Preoperative preparation}

Normal saline was used to clean and wash the intestines for 7-10 days, and oral antibiotics were taken for 3 days before the operation to prepare the digestive tract.

\section{Surgical method}

After successful anaesthesia, each patient was placed in a flat position; routine surgical field disinfection was performed, and the lower extremity was aseptically clothed by package isolation. The pneumoperitoneum needle was injected into the abdominal cavity at the lower edge of the umbilical part, and the artificial pneumoperitoneum was established by slowly injecting $\mathrm{CO} 2$ gas. The pneumoperitoneum pressure was generally maintained at 1.2-1.6 $\mathrm{kPa}$. After the pneumoperitoneum needle was removed, the $5 \mathrm{~mm}$ inner diameter laparoscopy was placed at the puncture point, and the operation forceps were placed at the intersection of the outer edge of the left rectus abdominis muscle and the umbilical horizontal line and the right lower abdomen. The extent of intestinal lesions was examined under laparoscopy to determine the spasmodic segment and the distal and proximal transitional segment. The serous muscle tissue of the upper rectum was cut and sent for intraoperative frozen biopsy. The pathological report showed that the submucous and intermuscular ganglion cells were diagnosed as "congenital megacolon". The area from the lesion section and the distal end to the upper section of the rectum was retroperitoneal to $1-2 \mathrm{~cm}$, the proximal end to the external appearance was soft, and the size of the intestinal tube was close to that of the normal intestinal tube. The proximal biopsies were taken from the proximal normal intestine. The sarco-muscular tissue of the segment was cut and sent for intraoperative frozen biopsy. The submucous and intermuscular ganglion cells were reported pathologically. After fully releasing the spleen curvature of the colon, the left side of the peritoneum, the lateral ligament of the descending colon, the lateral ligament of the sigmoid colon, and the proximal part of the examination could be stretched to the pelvic floor without tension. Then, we drained the gas from the abdominal cavity and transferred it to anal surgery. On the dentate line of the rectum, the posterior wall was $0.8 \mathrm{~cm}$, the anterior wall was $1.5 \mathrm{~cm}$, the rectal mucosa was cut in the oblique ring, and the 
rectal mucosa was free to the proximal end to the abdominal cavity. We circularly cut the muscle sheath and resected the part of the rectum muscle sheath in the posterior wall with the $\mathrm{V}$ type. The intestines were pulled out from the abdominal cavity with no tension and no torsion. At the proximal end of the examination, the serous layer of the lower segment of the colon was sutured with the stump of the rectal muscle sheath with an absorbable line of 4-0. The colon was cut off at the $0.5 \mathrm{~cm}$ distal end of the anastomosis. Then, intermittent suturing was performed between the severed end of the colon and the rectal mucosa. The artificial pneumoperitoneum was established again, no active bleeding was found in the abdominal cavity, the pulled-out colon was not reversed, the pneumoperitoneum was stopped and the gas was discharged, and the abdominal incision was closed. The anus was placed in the anal canal after the operation.

\section{Postoperative management}

After recovery of intestinal function, anal exhaust and defecation, the children began to take fluids and gradually returned to a normal diet for 5 days, with intravenous administration of antibiotics for 3 days. Anal dilatation began 14 days after the operation, once a day, fixed for $15 \mathrm{~min}$ to $20 \mathrm{~min}$, increasing by 1 calibre (1 mm) every week, and lasted for 3 months until number 20 anal dilatation rods were used.

\section{Postoperative follow-up}

The children were followed up by telephone and the outpatient service. The follow-up time was one week, one month, three months, six months, one year after operation, and then every 1-2 years. Instances of constipation, faecal incontinence, defaecation, enteritis, etc., were recorded.

\section{Results}

All 186 children underwent laparoscopic surgery successfully, and none progressed to open surgery. The operative time was $3.1(2.3-4.6) \mathrm{h}$, the amount of bleeding was $5(2-12) \mathrm{ml}$, the postoperative time to eating was $50(30-76) h$, and the postoperative hospital stay time was 7 (5-10) days (Table 1). During hospitalization and follow-up, there were 49 patients with complications, including 1 patient with an anastomotic leakage, 1 with an anal stricture, 5 with constipation recurrence, 5 with dirty defecation, 22 with enterocolitis, and 15 with perianal erosion. There were no complications such as abdominal bleeding, abdominal infection, ureter injury, adhesive intestinal obstruction, anastomotic stricture, incontinence. The follow-up period was 3 months to 5 years, and the median follow-up time was 3.5 years. The patient with an anal stricture, which was caused by the lack of time for the parents to dilate the anus, was cured by correct and reasonable anal dilatation after 1 year. For the 5 patients with constipation recurrence, 2 were cured by conservative treatment, and 3 were cured by repeat open operation. The defecation symptoms of the 5 children with dirty defecation gradually disappeared with increasing age and long-term anal sphincter exercise training. Children with enterocolitis were cured after conservative treatment, such as anti-infective use, clean enemas, and probiotics. Children with perianal erosion were cured after strengthening perianal nursing, keeping the perianal skin dry and clean, 
protecting perianal skin with external drugs, taking oral intestinal convergent drugs and reducing stool moisture.

\section{Discussion}

Hirschsprung disease is a common congenital digestive tract malformation in children caused by the lack of ganglion cells in the submucous nerve cluster of the diseased intestinal canal and the muscle of the intestinal wall, and spasm is caused by the continuous contraction of intestinal smooth muscle, which results in a defecation disorder ${ }^{[12,13]}$. The main treatment is surgery, the purpose of which is to remove the diseased intestinal canal and pull the intestinal canal innervated by the normal nerve to the anus for anastomosis to maintain the normal function of the anal sphincter and achieve the purpose of continuity of digestive tract reconstruction ${ }^{[14]}$. There are many traditional surgical methods. Swenson ${ }^{[15]}$ first treated HD through abdominal and perineal surgery, and Duhamel and Rehbein were carried out later; all had a long operation time, more bleeding and a high incidence of postoperative complications ${ }^{[16-18]}$. In recent years, the one-stage radical Soave operation of the transanal megacolon ${ }^{[19]}$ has been widely carried out because laparotomy is avoided, and there is less trauma, less bleeding and rapid postoperative recovery, but it is only suitable for the short segment type and some infants with the common type of megacolon. Thus, for many children with the common type, long segment type, total colon type and total intestinal type, open surgery cannot be avoided ${ }^{[20,21]}$. The application of laparoscopy can solve the technical limitations of the Soave transanal megacolon and reduce the trauma of laparotomy, which shows its minimally invasive advantages.

The laparoscopic-assisted Soave operation has the following advantages. First, the surgical trauma is small. Second, a good visual field can improve the positive rate of diagnosis, and occult diseases in the abdominal cavity can be detected and disposed of at the same time. Third, the operation can fully free the intestinal tube, which ensures a good blood supply to the terminal intestinal tube and avoids ischaemia. Anastomosing the intestinal tube without tension can ensure the relative existence of the "rectum" and retain the faecal storage function of the "new rectum". Fourth, laparoscopic surgery significantly shortens the anatomical operation time of the anus, which can reduce the risk of sphincter traction injury or insufficient intestinal resection and has a good effect on defaecating function. Fifth, pulling the colon out through the anus under the direct vision of laparoscopy can avoid intestinal volvulus and the injury of mesenteric blood vessels and ensure that there is no tension and no torsion in the pulled-out colon.

Although laparoscopic surgery has many advantages, because of the small abdominal cavity in children, abdominal distension often affects the laparoscopic visual field, resulting in abdominal organ injury, defective intestinal tube judgement, and affecting the normal laparoscopic operation ${ }^{[22]}$. We took the following measures to reduce abdominal distension and the difficulty of the operation, which ensured a smooth operation and avoided or reduced the conversion to laparotomy. First, we chose an experienced anaesthesiologist to avoid prolonged mask oxygen supply and repeated tracheal intubation. If there is 
obvious gas accumulation in the gastric vesicle, we can properly adjust the position of the gastric tube, keep the gastric tube unobstructed, and expel the gas from the stomach. Second, insertion into the anal canal or adult gastric canal through the anus and inserting the narrow segment into the dilated segment was performed to discharge the intestinal gas. Third, the small intestine often accumulates gas dilatation in the total colon type megacolon. The epidural catheter can be inserted through the abdominal wall to the dilated small intestine to eliminate the gas in the dilated small intestine and eliminate abdominal distension. Through the above measures to eliminate abdominal distension, all the children in this study successfully completed laparoscopic surgery, and none were converted to open surgery.

Enterocolitis was the most common and serious postoperative complication of Hirschsprung disease, with an incidence of $2-33 \%[23,24]$. In this study, enterocolitis occurred in 22 patients $(11.9 \%)$. Some scholars believe that the occurrence of enterocolitis is related to incomplete colorectal obstruction ${ }^{[25]}$, but we have observed that although most of the children have a smooth operation, standard anal dilatation after the operation, and no obvious stricture or obstruction at the distal end of the colon, enterocolitis still occurs. Therefore, we believe that in addition to colon obstruction, it is important to have low immunity in the body or intestine, surgical trauma and an imbalance of intestinal flora. All the children in this study were cured after conservative treatment, and some of the children with recurrent enterocolitis recovered gradually with the advancement of age and improvement in immune function.

Dirty defecation is a common postoperative complication of Hirschsprung disease, and 15 patients $(8.1 \%)$ were in this group. The main reasons were injury to the anal sphincter or excessive traction of the anus during the operation. When the colon was pulled out of the anus, the anal sphincter was damaged, which led to dysfunction of the anal sphincter. The occurrence of some dirty defecation was also related to congenital anal sphincter dysplasia ${ }^{[26]}$. Therefore, the action in the perineum should be gentle during the operation to avoid excessive traction of the anus, and injury of the levator anal muscle should be avoided during laparoscopic free retroflexion of the intestine. In this study, the children with defecation were not found to have congenital dysplasia or congenital loss of the anal sphincter by MRI, and all were cured after exercise training of the anal sphincter.

The causes of recurrence of constipation after the operation are as follows $[27,28]: 1$. The rectum muscle sheath without ganglia was retained too long. There was no incision or an insufficient incision of the posterior wall of the rectal muscle sheath during the operation. 2 . The resection of diseased intestine was insufficient: from a long segment megacolon to a short segment or ultra-short segment megacolon. 3. Due to an improper operation, proximal intestinal injury, and ischaemia, the secondary ganglion cells were poorly bred. 4. During the operation, the abdominal cavity was widely separated, and the blood vessels were damaged, resulting in spasm caused by insufficient blood supply to the internal sphincter and anal stricture, which caused constipation recurrence. 5. Enterocolitis was also an important cause of constipation recurrence. In this study, 5 patients with constipation recurrence and 2 patients with conservative treatment improved after the operation. One patient had recurrent, persistent constipation after surgery, and radiography showed colon dilatation. Considering that the resection scope was not sufficient, we resected the dilated segment. One patient showed a long segment megacolon by frozen 
pathology; the whole colon was pathologically confirmed by paraffin after the operation, and the radical operation of extubation was performed again. There was one patient with Hirschsprung disease complicated with megacolon-like disease with insufficient intestinal resection, resulting in a recurrence of constipation, which was cured by reoperation. Therefore, the cause of abnormal defecation must be found after the operation. After excluding anastomotic stricture, it should be clear whether the development of the intestinal nerve was normal and whether it was complicated by megacolon. It was also suggested that the pathological diagnostic criteria and pathologists' experience should be emphasized in the radical resection of megacolon.

There are several limitations of our study. First, this was a single-centre study, and more research from multiple centres is mandatory to assess the effectiveness and complications of this technique in further studies. Second, this study was a retrospective review without a control group.

\section{Conclusion}

In conclusion, the laparoscopic-assisted Soave operation is a safe and feasible method for the treatment of Hirschsprung disease in children. The cosmetic results are impressive, and the follow-up results are promising.

\section{Declarations}

Ethics approval and consent to participate This study was approved by the ethics committee of Fujian Maternity and Child Health Hospital and strictly adhered to the tenets of the Declaration of Helsinki. In addition, all patients' guardians signed an informed consent form before the operation.

\section{Consent for publication Not Applicable}

Availability of data and materials The datasets used and analysed during the current study are available from the corresponding author on reasonable request.

Competing interests The authors declare that they have no competing interests.

Funding: No founding.

Authors' contributions LMK and FYF designed the study, collected theclinical data, performed the statistical analysis, participated in the operation, and drafted the manuscript. ZB, LY, LOM, BJX and WDM participated in the operation and revised the article. All authors read and approved the final manuscript.

Acknowledgements We highly acknowledge the contribution by the participating doctors: Chaoming Zhou, Liu Chen, Jianqin Zhang and Bin Guo.

\section{References}


1. Fleur DL,Boeckxstaens GE,Benninga MA.Symptomatology,pathophysiology, diagnostic work-up,and treatment of Hirschsprung disease in infancy and childhood.Curr Gastroentero Rep.2007,9(3):245-53.

2. Sowande OA, Adejuyigbe O.Ten-year experience with the Swenson procedure in Nigerian children with Hirschsprung's disease. Afr J Paediatr Surg.2011,8:44-48.

3. Zhang $X H$,Zhu TY,Zhang W.Efficacy of umbilical laparoscopy and conventional laparoscopy in the treatment of congenital megacolon.Chinese Journal of Applied Pediatrics.2013,28(5):395-397》

4. Zhu T,Feng J,Zhang W,et al.Subtotal colectomy with a single-incision laparoscopic surgery technique in children with long-segment Hirschsprung disease and allied disorders.Pediatr Surg Int.2013,29(2):197.

5. De la Torre L,Cogley K,Santos K,et al.The anal canal is the fine line between "fecal incontinence and colitis"after a pull-through for Hirschsprung disease.J Pediatr Surg.2017,52(12):2011-2017.

6. Langer JC,Minkes $₫ \mathrm{~K}$, Mazziotti MV,et al.Transanal one-stage Soave procedure for infants with Hirschsprung's disease.J Pediatr Surg.1999,34(1):148》

7. Smith BM,Steiner RB,Lobe TE.Laparoscopic Duhamel pullthrough procedure for Hirschsprung's disease in childhood. J Laparoendosc Surg.1994,4(4):273-276.

8. Georgeson KE,Fuenfer MM,Hardin WD.Primary laparoscopic pullthrough for Hirschsprung's disease in infants and children.J Pediatr Surg.1995,30 (7):1017-1020.

9. Craigie RJ,Conway SJ,Cooper L,et al.Primary pull-through for Hirschsprung's disease:comparison of open and laparoscopic-assisted procedures.J Laparoendosc Adv Surg Tech A.2007,17(6):809- 812》

10. Tomuschat C,Zimmer J,Puri P.Laparoscopic-assisted pull-through operation for Hirschsprung's disease:a systematic review and meta-analysi.Pediatric Surgery International.2016,32(8):1-7》

11. Fujiwara N,Kaneyama K,Okazaki T,et al.A comparative study of laparoscopy-assisted pull-through and open pull-through for Hirschsprung's disease with special reference to postoperative fecal continence.J Pediatr Surg.2007,42(12): 2071-2074.

12. Tang YF,Chen JG,An HJ,et al.High-resolution anorectal manometry in newborns: normative values and diagnostic utility in Hirschsprung's disease.Neurogastroent Motil.2014,26(11):1565-1572.

13. Yan Z,Poroyko V,Gu S,et alCharacterization of the intestinal microbiome of Hirschsprung's disease with and without enterocolitis.Biochem Biophys Res Commun. 2014,445(2):269-274】

14. Xia SL,Li XN,Li B.long-term follow-up report of the operation of Soave assisted by laparoscopy.Journal of Clinical Pediatric surgery.2015,14(6): 512-515区

15. Swenson O,Bill Resection of rectum and rectosigmoid with preservation of the sphincter for benign spastic lesions producing megacolon.Surgery.1948,24:212-220.

16. Duhamel B.Une nouvelle operation panle megacolon congenital labaisement retrorectal et transanal du colon of san application possible au traitement de queleques autres malformation.Presse Med.1956,64:2249-2250.

17. Rehbein F. Intraabdominalle resection oder rectosigmoidektomie (Swenson) bei der hirschsprungschen krankheit.Chirurg.1958,29:366-369. 
18. Soave F.A new surgical technique for treatment of Hirschsprung's Surgey.1964,56:1007-1014.

19. De la Torre-Mondragon L,Ortega-Salgado JA.Transanal one-stage Soave procedure for Hirschprung's J Pediatr surg.1998,33:1283-1286.

20. Ekema G,Falchetti D,Torri F,et al.Further evidence on totally transanal one-stage pull-through procedure for Hirschsprung's disease:a multicenter study.J Pediatr 2003,38:1434-1439.

21. De La Torre L,Langer JC.Transanal endorectal pull-through for Hirschsprung disease:technique,controversies pearls,pitfalls, and an organized approach to the management of postoperative obstructive symptoms.Semin Pediatri Surg.2010,19(2): 96-106》

22. Shi QF,Li XN.Laparoscopic assisted radical resection of Soave in the treatment of congenital megacolon in children and its prevention and treatment.JOURNAL OF LAPAROSCOPIC SURGERY.2013,18(03):177-182

23. Demehri FR,Halaweish IF,Coran AG,et al.Hirschsprung-associated enterocolitis: pathogenesis,treatment and prevention.J Pediatr Surg,2013,29:873-881.

24. Suita S,Taguchi T,leiri S,et al.Hirschsprung's disease in japan:analysis of 3852 patients based on a nationwide survey in 30 years.J Pediatr Surg.2005,40:197-201.

25. Sun YC,Liu YM.Progress in transanal radical resection of Hirschsprungundefineds disease.Journal of Clinical Pediatric surgery.2009,8(2):56-57ه

26. Pena A,Elicevik M,Levitt MA.Reoperations in Hirschs-prung disease.J Pediatr Surg.2007,42(6): 10081013.

27. Wei MF,Wu XJ,Yi B.Study on the causes of recurrence of constipation after radical resection of megacolon.Journal of Clinical surgery.2008,16(5):324-326.

28. Xiong $X$,Chen $X$,Wang $G$,et al.Long term quality of life in patients with Hirschsprung's disease who underwent heart-shaped anastomosis during childhood: A twenty-year follow-up in China. J Pediatr Surg 2015;50:2044-2047.

\section{Table}

Due to technical limitations, Table 1 is only available for download from the Supplementary Files section.

\section{Supplementary Files}

This is a list of supplementary files associated with this preprint. Click to download.

- table1.doc 